\title{
El papel de la transdisciplinaridad y de los SIG en inventarios base para elaborar políticas públicas de producción económica y sustentabilidad ambiental
}

\author{
O papel da transdisciplinaridade e dos SIG em inventários base para a \\ formulação de políticas públicas de produção econômica e \\ sustentabilidade ambiental
}

The role of transdisciplinarity and GIS in basic inventories to elaborate public policies of economic production and environmental sustainability

\author{
Lic. Armando Benito Brizuela ${ }^{1}$ \\ Dr. César Augusto Aguirre ${ }^{2}$
}

\begin{abstract}
Resumen
Después de siglos de uso y aprovechamiento de los recursos naturales, con el consiguiente deterioro y pérdida de la capacidad productiva, se produjo un impacto notable en las comunidades que dependen de los mismos. En la actualidad, se percibe que estos recursos son finitos y muchos son escasos. Siendo así, importa disponer de inventarios que sean útiles en la formulación de políticas sustentables. Esto implica que los relevamientos cuantitativos no son suficientes de tal manera que, para lograr para un abordaje sistémico, es necesario optar por otros enfoques superadores. En este trabajo se da cuenta de la aplicación de técnicas de teledetección y sistemas de información geográfica en relevamientos de bosque nativo, plantaciones forestales y citrus en la provincia de Entre Ríos, Argentina. Se reflexiona que el diálogo intercientífico con orientación transdisciplinaria puede perfeccionar estas investigaciones cuando son aplicadas para subsidiar la formulación de políticas públicas.
\end{abstract}

Palabras claves: recursos naturales, inventarios, sistemas de información geográfica, diálogo intercientífico.

\section{Resumo}

Após séculos de uso e aproveitamento dos recursos naturais, com o conseguinte deterioro e perda da capacidade produtiva, tem se produzido um impacto notável nas comunidades que dependem dos mesmos. Na atualidade, se percebe que estes recursos são finitos e muitos são escassos. Sendo assim, é importante dispor de inventários que sejam úteis na formulação de políticas sustentáveis. Isto implica que os estudos quantitativos não são suficientes de tal forma que, para atingir uma abordagem sistêmica, é necessário optar por outras abordagens superadoras. Neste trabalho se evidencia a aplicação de técnicas de teledetecção e sistemas de informação geográfica em estudos de inventário da mata nativa, plantações florestais e cítricas na província de Entre Ríos, Argentina. Acredita-se que o diálogo intercientífico com orientação transdisciplinaria pode aperfeiçoar essas pesquisas quando elas subsidiam a formulação de políticas públicas.

Palavras-Chave: diálogo intercientífico, inventários, recursos naturais, sistemas de informação geográfica

\section{Abstract}

\footnotetext{
${ }^{1}$ Licenciado en Ciencias Meteorológicas (UBA); Profesor Titular de la Universidad Nacional de Entre Ríos UNER; Investigador Adjunto del CONICET; Paraná, Entre Ríos, Argentina; armando.brizuela@ gmail.com.

${ }^{2}$ Doctor en Ingeniería (Université Claude Bernard, Francia). Profesor Titular de la Universidad Nacional de Entre Ríos - UNER; Investigador Adjunto del CONICET; Paraná, Entre Ríos, Argentina; cesaraguirredalotto@gmail.com.
} 
Centuries of using and exploiting natural resources and their consequential deterioration and loss of productive capacity resulted in a notable impact on the communities that depend on them. Currently, these resources are finite, and many are scarce. As a result, it is important to maintain inventories that are useful in formulating sustainable policies. Quantitative studies are not sufficient. Thus, to achieve a systemic approach, it is necessary to pursue other strategies. This work reveals the application of remote sensing techniques and geographic information systems in studies of native woodlands and forest and citrus crops in the province of Entre Rios, Argentina. Interdisciplinary dialogues with a transdisciplinary orientation can improve these investigations when they undergird public policy formulation.

Keywords: inter-scientific dialogue, inventories, geographic information systems, natural resources.

\section{Introducción}

Un problema común - con otros sectores relacionados con los recursos naturales - es la falta de datos confiables, exactos y actuales sobre la cantidad y localización de los recursos, necesarios para la formulación de las políticas públicas. En esta situación es indudable que la teledetección aplicada y los sistemas de información geográfica constituyen una herramienta eficiente para apoyar los relevamientos y obtener en forma expeditiva datos de calidad y exactitud.

Los recursos naturales, son considerados generalmente como bienes libres. El aire, el agua y la tierra son considerados de esta manera y se diferencian de los bienes económicos a los que se les asigna un valor económico. En este sentido resulta paradójico que los llamados recursos naturales por un lado hacen posible satisfacer las necesidades fundamentales de nuestra sociedad y por otro no son convenientemente valorizados, preservados ni reconocidos. "Su valorización monetaria es difícil y políticamente delicada; sin embargo, sería la única manera de evitar su desaparición, si se asegura que los precios de los recursos naturales reflejen efectivamente su utilidad y su escasez relativa" (ARGENTINA, 1995, p.198).

Según Herz (1993, p.83),

Los estilos de desarrollo predominantes se han caracterizado por una explotación masiva e irracional de los recursos naturales. El crecimiento económico ha sido erróneamente confundido con desarrollo, distorsionando indicadores, parámetros, estrategias y políticas de planificación. Muchos países han visto crecer sus economías sin que ello haya implicado la superación de los niveles de calidad de vida, llegando a extremos de una creciente degradación social y pérdida de valores fundamentales que posibilitan una armónica convivencia social.

El artículo 41 de la Constitución Argentina reformada en 1994 proclama que:

Todos los habitantes gozan del derecho a un ambiente sano, equilibrado, apto para el desarrollo humano y para que las actividades productivas satisfagan las necesidades presentes sin comprometer las de las generaciones futuras, y tienen el deber de preservarlo. El daño ambiental generará prioritariamente la obligación de recomponer, según establezca la ley. Las autoridades proveerán a la preservación del patrimonio natural y cultural y de la diversidad biológica y a la información y educación ambiental (ARGENTINA, 1994). 
En este trabajo se hace referencia a inventarios de bosque nativo, forestal y citrus realizados en la provincia de Entre Ríos señalando que estos son fundamentales para la formulación de programas y planes sociales. Para realizarlos se utilizaron herramientas de sistemas de información geográfica. Finalmente, señalamos el desafío de la transdisciplinariedad y el diálogo intercientífico en la ejecución y monitoreo de las políticas públicas, además de la participación universitaria y ciudadana en cada uno de estos procesos.

\section{Bosques nativos y selvas ribereñas de Entre Ríos}

A principios del siglo XX la superficie cubierta con montes nativos de la Argentina era de 106 millones de hectáreas (ha) de las cuales en la actualidad quedarían menos de 30 millones de ha. Durante el año 2003, una vez comprobada la alta tasa de deforestación y sobre la base de las garantías de la Constitución Nacional, el gobierno de Entre Ríos declaró la emergencia ambiental de los bosques nativos en la provincia mediante el decreto $\mathrm{N}^{\circ} 4519 / 03$ SEPG, el cual estableció severas sanciones para quienes practiquen la tala rasa dentro del territorio provincial.

Al año siguiente, el nuevo gobierno provincial de Entre Ríos derogó esta normativa mediante el Decreto No 1317/04, por lo cual el Foro Ecologista de Paraná promovió la Acción de Amparo Ambiental y Acción de Ejecución contra el Superior Gobierno de la Provincia de Entre Ríos en los términos de los artículos 41 y 43 de la Constitución Nacional y de los artículos 2, 3 inc. c). 4, 6 y cap. $\mathrm{V}$ de la Ley $\mathrm{N}^{\circ} 8369$, pretendiendo se declare la inconstitucionalidad del Decreto $\mathrm{N}^{\mathrm{o}} 1317 / 04$ y se ordene al Poder Ejecutivo el mantenimiento de la emergencia ambiental del bosque nativo y la diversidad biológica que lo conforma y que se apruebe un Plan de Preservación, Recomposición y Sustentabilidad del Bosque Nativo de Entre Ríos. La justicia hizo lugar a la acción de amparo y surgieron instancias de conciliación. Luego de varios encuentros las entidades de la producción, el gobierno y la organización ecologista acordaron el establecimiento de un sistema que compatibilice el accionar de todas las entidades de la producción.

Muñoz et al. (2003), utilizando imágenes de satélite y sistemas de información geográfica, realizaron un inventario con el objeto de obtener la superficie ocupada por bosques nativos y selvas ribereñas en la Provincia de Entre Ríos. Posteriormente, en el año 2007, fue sancionada la Ley nacional N²6.331 de 'Presupuestos mínimos de protección ambiental de los bosques nativos' que establece las categorías de conservación de los bosques nativos. La provincia de Entre Ríos adhiere a dicha ley en todos sus términos: 
Las categorías de conservación de los bosques nativos son las siguientes: - Categoría I (rojo): sectores de muy alto valor de conservación que no deben transformarse. Incluirá áreas que por sus ubicaciones relativas a reservas, su valor de conectividad, la presencia de valores biológicos sobresalientes y/o la protección de cuencas que ejercen, ameritan su persistencia como bosque a perpetuidad, aunque estos sectores puedan ser hábitat de comunidades indígenas y ser objeto de investigación científica. - Categoría II (amarillo): sectores de mediano valor de conservación, que pueden estar degradados pero que a juicio de la autoridad de aplicación jurisdiccional con la implementación de actividades de restauración pueden tener un valor alto de conservación y que podrán ser sometidos a los siguientes usos: aprovechamiento sostenible, turismo, recolección e investigación científica.- Categoría III (verde): sectores de bajo valor de conservación que pueden transformarse parcialmente o en su totalidad aunque dentro de los criterios de la presente ley (ARGENTINA, Ley 26.331).

Durante los años de elaboración y aprobación de las leyes, nacionales y provinciales, hubo una gran movilidad y participación social - sobre todo de instituciones y organizaciones ecologistas-. Sabattini et al. (2009) elaboraron los mapas delimitando las tres categorías para los departamentos del centro-norte de Entre Ríos utilizando imágenes satelitales y herramientas de los sistemas de información geográfica, como en el relevamiento anterior. Desde la Facultad de Ciencias Agropecuarias en su página web se publicaron los informes, de la zonificación de los bosques nativos según las categorías de conservación, que incluyen mapas detallados y superficie por departamento (Figura 1). 
RELACult - Revista Latino-Americana de Estudos em Cultura e Sociedade

Revista Latinoamericana de Estudios en Cultura y Sociedad | Latin American Journal of Studies in Culture and Society V. 03, ed. especial, dez., 2017, artigo $n^{\circ} 595$ | relacult.claec.org | e-ISSN: 2525-7870

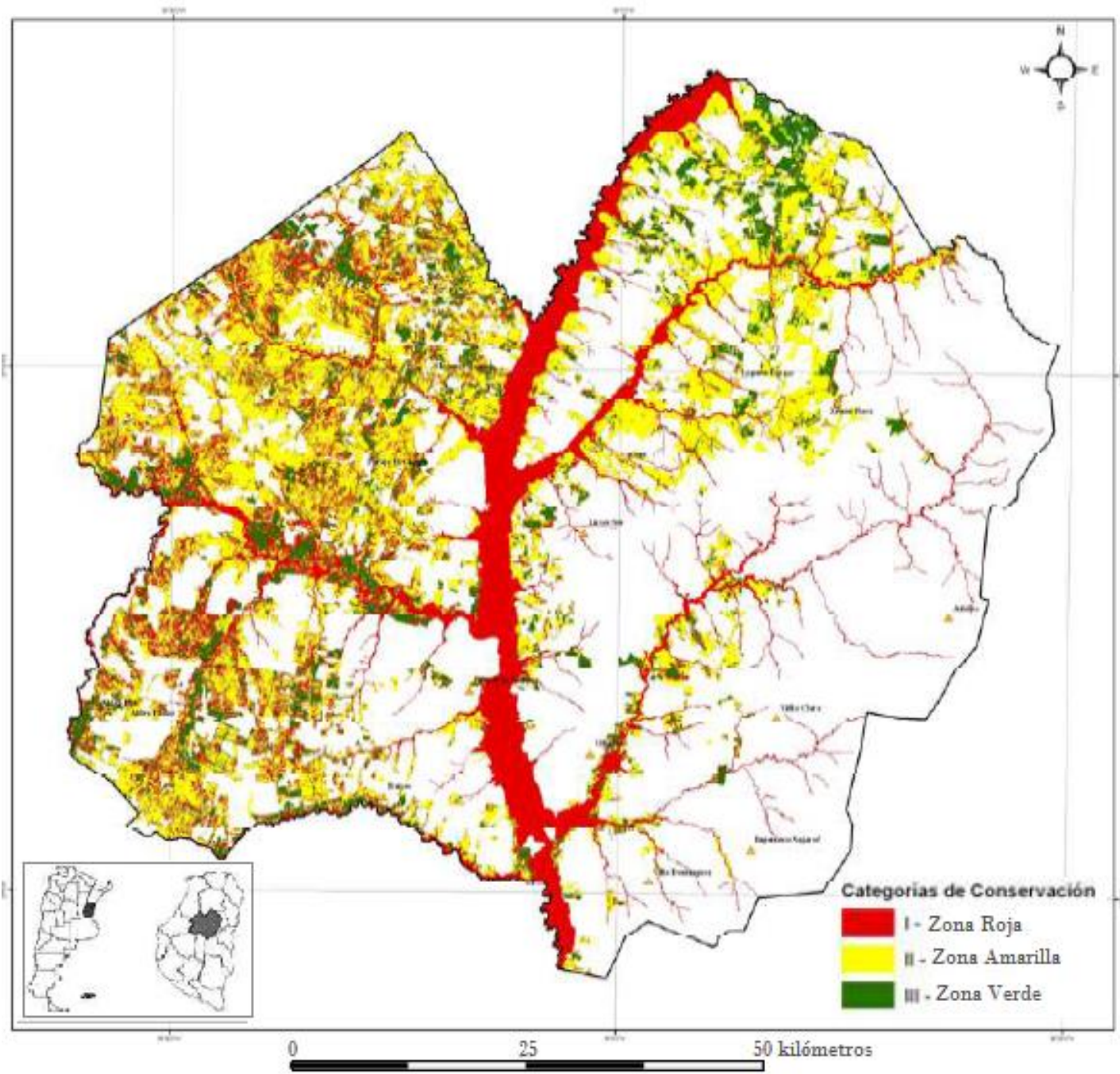

Figura 1 - Categorías de conservación de bosques nativos en el Departamento Villaguay, Entre Ríos (Arg.).

Fuente: Sabattini et al. (2009, p.16)

\section{Plantaciones de eucaliptos y pinos}

El conocimiento de la localización y cuantificación - con exactitud - del recurso forestal y la posibilidad de disponer de esta información en tiempo real, son aspectos relevantes en el orden provincial, nacional y aún internacional. La importancia del sector fue puesta de manifiesto por el Proyecto Forestal de Desarrollo tanto en las posibilidades que ofrecen los suelos, del norte de la provincia, como en el inventario de plantaciones forestales (ARGENTINA, 2001).

Para realizar un relevamiento de bosques implantados en los departamentos del este de Entre Ríos se utilizaron imágenes del satélite Landsat 7 ETM + de diciembre de 2002 y enero 2003 (BRIZUELA et al., 2004). El trabajo posibilitó cuantificar y localizar todos los lotes 
ocupados por plantaciones de eucaliptos y pinos, así como mostrar aplicaciones de sensores remotos y SIG en la obtención de este tipo de inventarios. Ese estudio mostró que en los departamentos de Federación, Concordia y Colón la superficie de eucaliptos superaba las 75.000 ha y la de pinos era cercana a las 8.000 ha (Figura 2).

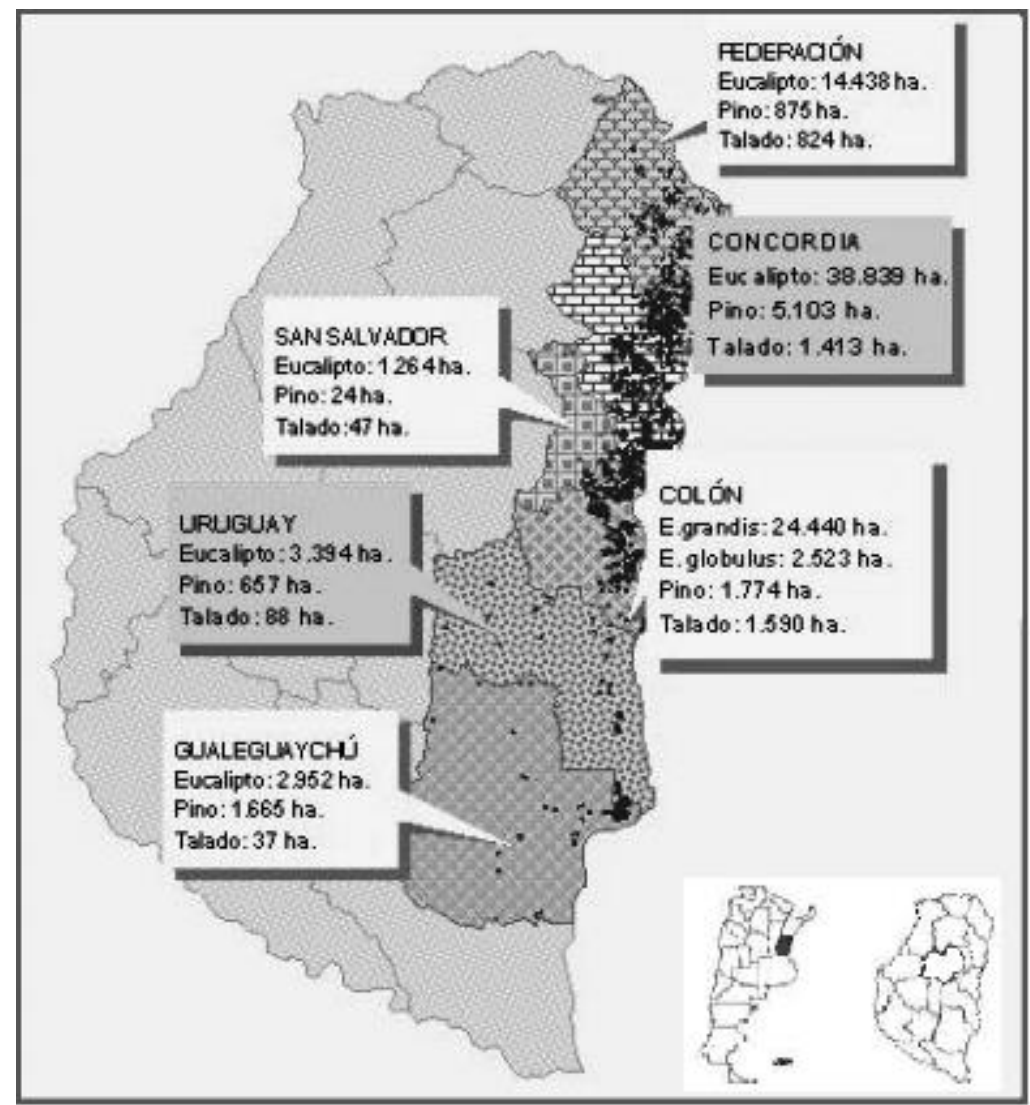

Figura 2 - Distribución de las bosques implantados en la provincia de Entre Ríos (Arg.) -diciembre 2002, enero 2003-.

Fuente: Brizuela et al. (2004, p. 158)

La cadena foresto-industrial integra diversos procesos, desde la producción hasta la comercialización del producto final. Esta diversidad de actividades implica también un número importante y diverso de actores, que incluye viveristas, productores y empresas forestales, profesionales, empresas de servicios y proveedoras de insumos, industrias forestales, operarios forestales e industriales así como instituciones de ciencia y tecnología, y entidades gremiales del sector (ARGENTINA, 2016). Sin embargo, los distintos gobiernos provinciales y nacionales no pusieron en práctica políticas para el sector que mejoren significativamente el bienestar del conjunto de productores y trabajadores con menores recursos económicos. En este sentido, las herramientas de investigadores en ciencias sociales y estudiosos de los diálogos intercientíficos e interculturales son muy valiosas para el proceso de construcción de diagnósticos situacionales y políticas públicas, como veremos más 
adelante.

Vale la pena registrar que la industrialización de los productos forestales puede significar conflictos de intereses económicos y ambientales. Por ejemplo la instalación de pasteras principalmente para elaborar pasta celulosa, que se llevó adelante con autorización del gobierno uruguayo, produjo un conflicto entre Uruguay y Argentina que incluyó litigios ante organismos internacionales. Si bien gran parte de la población de Gualeguaychú (Entre Ríos), empresarios vinculados al turismo y organizaciones ambientalistas se opusieron a la instalación de las plantas sobre el río Uruguay, con movilizaciones y cortes de rutas, una de las plantas finalmente se estableció y aún hay controversias en cuanto a efectos económicos y ambientales de este tipo de emprendimientos.

\section{Censo citrícola de Entre Ríos}

La citricultura tiene un alto impacto en la economía regional, aportando ingresos genuinos y demandando recursos humanos necesarios para el desarrollo y crecimiento de la cadena. Los establecimientos productivos en general son pequeños o de poca envergadura y se caracterizan por ser un sistema de producción de tipo familiar con gran capacidad de trabajo (CFI, 2016).

GARCIA (2017) analiza la vinculación entre la agricultura familiar (AF) y agroindustrias en el corredor citrícola del Río Uruguay Medio y fruticultura de Valles Patagónicos. Realiza una caracterización detallada de los actores y agentes presentes en la fase primaria y en la comercialización. Considera también aspectos de mercado mundial y sus relaciones con la reestructuración productiva local. Afirma que:

la producción familiar se transforma, tendiendo en algunos casos a especializarse en algún cultivo de renta y reduciendo su diversidad productiva, siendo posible la estabilización de la subordinación productiva debido a que la AF se transforma en simple proveedor de materia prima hacia la agroindustria, relegando otros cultivos y/o actividades.

Este diagnóstico, válido para el corredor citrícola de Entre Ríos, pone en evidencia la necesidad de diseñar y poner en práctica políticas públicas para el sector que contemplen todos los aspectos de sustentabilidad social, ecológica y económica. Dichas políticas no están presentes en la provincia y constituyen un desafío principalmente a los funcionarios de gobierno, así como a los investigadores, universidades y empresas para proponer respuestas viables que signifiquen mejorar la situación de los productores familiares.

En el año 2003/04 la Federación del Citrus de Entre Ríos (FECIER) conjuntamente 
con las Asociaciones de citricultores y empacadores de la región con el apoyo de los productores de los departamentos de Federación, Concordia, Federal y Colón realizaron un relevamiento de las plantaciones de citrus. En esa oportunidad se llevó a cabo un censo apoyado con datos de sensores remotos y tratamiento de los datos en un entorno SIG. Luego de más de una década la FECIER consideró necesario actualizar los datos para lo cual se realizó el censo citrícola 2015-2016. Los resultados de superficie citrícola, número de quintas y número de productores, por departamentos, se indican en la Tabla 1 (Censo 2003/2004) y en la Tabla 2 (Censo 2015/2016).

El número de productores (cerca de 1800) y de quintas (cerca de 2350) es similar en ambos censos. Sin embargo en el departamento Concordia se redujo la cantidad de productores en un $45 \%$ y de quintas en $40 \%$.

Tabla 1. Superficie de citrus, número de quintas y número de productores en Entre Ríos según el Censo 2003-2004.

\begin{tabular}{|c|c|c|c|c|c|c|}
\hline Dpto. & $\begin{array}{c}\text { Superficie } \\
\text { (ha) }\end{array}$ & $\%$ & $\mathrm{~N}^{\mathrm{o}}$ de quintas & $\%$ & $\mathrm{~N}^{\mathrm{o}}$ de productores & $\%$ \\
\hline Colón & 566,6 & 1,33 & 8 & 0,33 & 6 & 0,34 \\
\hline Concordia & 13977,5 & 32,84 & 563 & 23,51 & 446 & 24,97 \\
\hline Federación & 27996,9 & 65,78 & 1817 & 75,87 & 1327 & 74,30 \\
\hline Federal & 20,1 & 0,05 & 7 & 0,29 & 7 & 0,39 \\
\hline Total & 42561,1 & 100 & 2395 & 100 & 1786 & 100 \\
\hline
\end{tabular}

Fuente: Brizuela et al. (2016, p. 7)

Tabla 2. Superficie de citrus, número de quintas y número de productores en Entre Ríos según el Censo 2015-2016.

\begin{tabular}{|c|c|c|c|c|c|c|}
\hline Dpto. & $\begin{array}{c}\text { Superficie } \\
\text { (ha) }\end{array}$ & $\%$ & $\begin{array}{l}\mathrm{N}^{\circ} \text { de } \\
\text { quintas }\end{array}$ & $\%$ & $\mathrm{~N}^{\mathrm{o}}$ de productores & $\%$ \\
\hline Colón & 117 & 0,4 & 4 & 0,2 & 3 & 0,2 \\
\hline Concordia & 6891 & 18,9 & 343 & 14,6 & 241 & 12,8 \\
\hline Federación & 29379 & 80,7 & 2005 & 85,2 & 1634 & 87,0 \\
\hline Total & 36387 & 100 & 2352 & 100 & 1878 & 100 \\
\hline
\end{tabular}

Fuente: Brizuela et al. (2016, p. 7-8)

La escasa rentabilidad, elevados costos de producción, enfermedades de la fruta y 
diversidad de cultivos son algunas de las causas que explican el cambio. También en Concordia fue significativo el número de quintas abandonadas. Este proceso se acentuó en los últimos años y refleja claramente un problema que afecta concretamente a productores familiares. Diferente es el caso de Federación, que tiene una mayor organización de sus productores y mejor preparación para la comercialización de sus productos lo que se traduce en un aprovechamiento intensivo de la superficie utilizada y dedicada al citrus. Es decir, con más información, organización, articulación y preparación especializada de los productores familiares, se obtienen mejores resultados en términos económicos y de sustentabilidad ambiental.

\section{Reflexiones desde la opción interdisciplinaria o transdisciplinaria}

En los tres casos anteriores se da cuenta de un aspecto principalmente cuantitativo en el que los relevamientos o inventarios son logrados aplicando técnicas y métodos propios de las Ciencias de la Tierra que responden, en gran medida, a los paradigmas de la ciencia convencional. Sin embargo en años recientes en las universidades argentinas y en los organismos de ciencia y tecnología se observa una búsqueda de la interdisciplinariedad científica como un desafío que les incumbe.

Promover reflexiones, sobre la base de estas ideas, en el ámbito de nuestra universidad no es tarea sencilla - por el sistema mismo, la formación académica, estructura, organización, forma de gobierno, etc. - pero siempre es posible alimentar la expectativa de sumar logros.

Según Mignolo (2009, p. 13) "la epistemología occidental escondió su propia geo y corpo-política”, las Ciencias de la Tierra no estarían exentas por cuanto, según el autor, “... el conocedor siempre está implicado corpo y geopolíticamente en lo conocido" (p. 14), aunque no se explicita. Por eso es importante pensar desde estas ciencias si no es necesario ampliar o completar la desobediencia epistémica y asumir que

La tarea del pensamiento decolonial es develar los silencios epistémicos de la epistemología occidental y afirmar los derechos epistémicos de las opciones decoloniales racialmente devaluadas, para permitir, desde el silencio, construir argumentos que confronten a aquéllos que toman a la "originalidad" como el criterio máximo para el juicio final (MIGNOLO, 2009, p. 14).

También Mignolo menciona "el reclamo de que el hacer-conocimiento para bien estar más que para controlar y administrar poblaciones por interés imperial, vendrán de experiencias y necesidades locales.." (p. 38), señalando un camino para las nuevas investigaciones. 
Un aspecto que también merece atención es la carencia de inclusión explícita de las personas en los estudios, además de las cosas u objetos que podrían proporcionar acercamiento al buen vivir. El humano es un integrante de la naturaleza y esta puede ser considerada como una red dinámica de relaciones interconectadas que lo incluye. Se puede considerar como una carencia o debilidad de las investigaciones de las ciencias de la tierra cuando priorizan los aspectos materiales, cuantitativos y desestiman o desconocen los de orden cualitativo.

Los tres casos comentados, como muchos otros trabajos de investigación en Ciencias de la Tierra, carecen de complementariedad disciplinaria. El uso de los recursos naturales y aprovechamiento durante siglos ha llevado a un creciente deterioro de los mismos, a la pérdida de su capacidad productiva y produjo un impacto notable en las comunidades que de alguna manera dependen de ellos. En las últimas décadas se gana conciencia sobre la necesidad y conveniencia de conservar los recursos que se consideraban inagotables pero que ahora se percibe que son finitos y muchos son escasos. En consecuencia desde diferentes puntos de partida y en distintas direcciones vienen presentándose ideas y acciones tendientes a una mejor valoración de los recursos naturales, se trata de conservarlos, protegerlos, hacer uso y aprovechamiento sustentables.

Una alternativa para modificar el esquema tradicional de la investigación y desarrollo es la transdisciplinariedad. Para eso habrá que tener en cuenta que:

[...] aún falta encontrar puntos de articulación entre teoría y práctica alrededor de este tema. Y no es cuestión de si es un concepto, enfoque, o técnica investigativa la que viaja de una disciplina a otra, o si se trata de una práctica investigativa realizada por profesionales de varias disciplinas (con y sin articulación evidente), el problema es mucho más complejo, es cuestión de entender que cuando se trabaja con objetos de investigación (estudio) se debe dar cuenta (formalizar) de los alcances de estos intercambios, es entender que se está trabajando con construcciones que trascienden lo disciplinar. Tal vez por eso es que muchos trabajos de investigación realizados a nivel universitario por investigadores e investigadoras en formación, e incluso de trayectoria (maestría y doctorado), pierden toda pertinencia al tocar la dimensión práctica, pues se formulan desde las temáticas que se trabajan y no desde la construcción de un objeto de investigación. Aún hay mucho que reflexionar y construir (PEÑUELA VELÁSQUEZ, 2005, p.73).

Para ir más allá de lo disciplinar, comprender y aplicar saberes y tecnologías en forma integral, holística y transdisciplinar; se puede aprovechar experiencias en las que:

[...] fue necesario ampliar los conocimientos más allá de lo técnico agronómico y complementarlos con las ciencias sociales, humanas y económicas, desarrollando un enfoque holístico y transdisciplinar que estableció un diálogo permanente al interior del equipo y con las comunidades con las que se interactuaba, en busca de alternativas cada vez más sustentables, además para aportar nuevos paradigmas a las ciencias y las visiones de desarrollo (Rist et al., 2010, p.16). 
En este sentido se destaca que las actividades comentadas se desarrollan en un ámbito urbano-rural, de Entre Ríos, donde ocurren transformaciones a lo largo del tiempo que implican una realidad cambiante especialmente en la dinámica funcional en el vínculo espacial urbano y rural. Aquí los sistemas de información geográfica facilitan la comprensión de las transformaciones y procesos. Al respecto Picciani (2016) sostiene:

\begin{abstract}
El actual proceso productivo y en este caso la expansión agropecuaria crea lógicas que son el motivo de acciones selectivas y jerárquicas materializadas en el territorio las cuales no pueden ser explicadas desde una mirada acrítica, porque justamente no serán producto de acciones homogéneas sino muy por el contrario serán creaciones fruto de contradicciones, de diferentes formas de producción, de vida, de diversas racionalidades e intencionalidades, acciones, formas, herencias materiales y técnicas que conviven en el mundo. Se crean nodos urbanos regionales que actúan de plataforma para la expansión de este modo de producción, pero las jerarquías urbanas creadas con anterioridad no necesariamente se hallan involucradas. Aquí está el desafío del investigador, el poder comprender una composición compleja de fusiones y relaciones, de eventos y de normas, de contenidos y de formas. Solo una visión totalizadora sobre una situación espacializada constituirá un buen método para explicar la realidad, por lo tanto entender el vínculo urbano y rural hoy implica empirizar los eventos de acuerdo a su tiempo, es decir considerarlos como el producto de una yuxtaposición, de una superposición de aconteceres, donde órdenes verticales y horizontales dan lugar a la creación de nuevos eventos y por lo tanto de nuevas formaciones socio-espaciales, serán los fenómenos técnicos los que explicarán cómo, dónde, por qué, por quién y para qué el territorio es usado (p. 25).
\end{abstract}

La agricultura familiar, las pequeñas y medianas empresas -citrícolas, forestales, agroganaderas- como se comentó representan, para una parte de la población de Entre Ríos, una forma de incorporarse al sistema de supervivencia por la obtención de productos de consumo propio o proveyendo al comercio de alimentos para consumo local o regional. Esta no es una condición exclusiva de esta provincia, ni de Argentina, pues según Schejtman (2008) en varios países de América Latina:

la agricultura familiar es un importante proveedor de muchos de los alimentos básicos de consumo popular [..]. El creciente interés y preocupación por el desarrollo de la agricultura familiar está estrechamente vinculado al tema de la pobreza rural, más que al del desarrollo de la agricultura como tal.[..]. Los agricultores familiares, al igual que otras familias rurales, constituyen un grupo particularmente afectado por la pobreza rural. [..].El Consejo del MERCOSUR viene impulsando la red especializada en agricultura familiar (REAF), como un órgano dependiente que tiene entre sus objetivos la identificación y fortalecimiento de políticas públicas diferenciadas para la agricultura familiar así como la coordinación entre los Estados en la materia (p. 12-14).

La asimetría entre los productores rurales y las empresas agroindustriales plantea a los gobernantes un escenario sensible donde deben formular e implementar políticas públicas que atiendan las necesidades de la población y eviten la exclusión social. En un contexto más amplio Sabourin (2014) presenta un análisis teórico y metodológico sobre políticas públicas 
relacionadas con la agricultura familiar en América Latina y el Caribe donde señala que el apoyo financiero, establecido por las políticas para el sector familiar, sigue siendo muy inferior al que recibe la agricultura empresarial en todos los países del estudio (p.24).

Como se percibe la formulación y puesta en práctica de políticas públicas alcanza dimensiones, relaciones e interacciones complejas. El conocimiento cuantitativo preciso de los recursos con la información de ‘¿qué?’, ‘¿cuánto?’, ‘¿dónde?’ que ofrecen los sistemas información geográfica, describe parcialmente la realidad de los fenómenos actuales. Esto exige otra forma de pensar, valorar e interpretar la realidad actual desde una perspectiva más amplia como propone el enfoque de la transdisciplinariedad que busca superar la fragmentación del conocimiento más allá de lo disciplinar, de la epistemología convencional y de la visión interdisciplinaria acotada. También implica participar en procesos que den lugar a nuevos saberes integrando y transformando diferentes miradas científicas para tratar los problemas complejos de la realidad.

\section{Conclusión}

La transdisciplinariedad es una opción que posibilita ir más allá para lograr un conocimiento totalizador e integrador en condiciones de dar respuesta los interrogantes de la ciencia actual para explicar cómo, dónde, por qué, para quién, cuándo, por quién y para qué es usado el territorio.

En síntesis, nuestra universidad puede iniciar un nuevo recorrido de diálogo intercientífico desde una perspectiva interdisciplinaria y avanzar a la transdisciplinaria de tal manera que sea posible concretar las tareas de 'conocer', 'des-estructurar conceptos' y 'tratar de manera sistémica' otros conocimientos, en especial, frente a las complejas demandas de las políticas públicas contemporáneas.

\section{Referencias}

ARGENTINA. Constitución de la Nación. Santa Fe-Paraná. Editorial La Ley, 1994. SECRETARÍA DE AGRICULTURA. GANADERÍA y PESCA (SAGyP) y CONSEJO FEDERAL AGROPECUARIO (CFA). El deterioro de las tierras en la República Argentina. Alerta Amarillo, 1995. 277 pp.

. SAGPyA, Inventario Nacional de Plantaciones Forestales, Proyecto Forestal de Desarrollo (PIA 33/97) 1, 2001. 63 pp.

. Ministerio de Agroindustria. Política Forestal Argentina. Entre Ríos: 
Cluster Forestal de la Costa del Río Uruguay, 2016. Disponible en: $<$ http://politicaforestalargentina.blogspot.com.ar/p/aportes-para-una-politica-forestal.html>.

Acceso en: 21 Abril 2017.

BRIZUELA, A. B., AGUIRRE C. A. y SCATTONE G. Censo citrícola 2015-2016. Federación del Citrus de Entre Ríos y Facultad de Ciencias Agropecuarias -UNER-, 2016, 19 p. Disponible en: http://www.noticias.uner.edu.ar/public/attached/1071714702261.pdf Acceso en: 20 Abril 2017

BRIZUELA, A. B.; MILERA, S.; MESTRES, J. Plantaciones de Eucaliptos y Pinos en los departamentos del este de Entre Ríos. INSUGEO, Miscelánea 12, 2004. p. 153-160.

Consejo Federal de Inversiones (CFI). Informe Final: Censo Provincial Citrícola 2015/2016. Concordia, Entre Ríos, 2016. 49 p.

GARCIA, A. O. Agricultura Familiar y Agroindustrias: subordinación productiva. Los casos de la citricultura y la fruticultura de Argentina (2003-2016). Redes, 2017, vol. 22, no 2. p. 374-400.

HERZ. C. Pobreza y Medio Ambiente. Resúmenes del Congreso Forestal Argentino y Latinoamericano. Comisión III. Protección Ambiental y Bosques, Asociación Forestal Argentina V 3, 1993. p. 83-96.

MIGNOLO, Walter. Desobediencia Epistémica (II), Pensamiento Independiente y Libertad De-Colonial. Otros Logos, Revista de Estudios Críticos, 2009, vol. 1, no 1, p. 8-42.

MUÑOZ, J. D. D.; MILERA, S.; ROMERO, C.; BRIZUELA, A. Bosques nativos y selvas ribereñas en la provincia de Entre Ríos. Temas de la biodiversidad del litoral fluvial argentino. INSUGEO Miscelánea, 2005. p. 169-182.

PEÑUELA VELÁSQUEZ, A. La transdisciplinariedad: Mas allá de los conceptos, la dialéctica. Andamios, vol. 1, no 2, 2005. p. 43-77. Disponible en: $\langle$ http://www.scielo.org.mx/scielo.php?pid=S1870-00632005000300003\&script=sci arttext $>$.

Acceso en: 21 Abr. 2017.

PICCIANI, Ana Laura. Discusiones teóricas sobre la dinámica funcional en el vínculo espacial urbano y rural. Pampa (Santa Fe), 2016, no 14, p. 9-28.

RIST, S.; DELGADO BURGOA, F.; ESCOBAR VASQUEZ, C. El desarrollo endógeno sustentable como interfaz para implementar el Vivir Bien en la gestión pública boliviana. AGRUCO. CAPTURED. PLURAL, 2010. 62 p.

SABATTINI, R. A.; LEDESMA, S.; BRIZUELA, A.; SABATTINI, J. A.; FONTANA, E.; DIEZ, J. M.; SABATTINI I. Informe 4. Zonificación de los bosques nativos en el Departamento Feliciano (Entre Ríos) según las categorías de conservación. Convenio FCADGRN-Sec. Producción-, Entre Ríos, Argentina, 2009. 30 p.

SABOURIN, E. et al. Red políticas públicas y desarrollo rural en América latina. Informe de actividades 2014. Programación 2015. 2014. 298 p. 
SCHEJTMAN, A. Alcances sobre la agricultura familiar. Diálogo Rural Iberoamericano, San Salvador, Documento de Trabajo N²1. Programa Dinámicas Territoriales Rurales. Rimisp, Santiago, Chile. Septiembre 2008. 45 p. 